\title{
VIH, dislipidemias y perspectivas de su evolución
}

\author{
HIV, dyslipidemias and perspectives of its evolution
}

Hugo Mendieta Zerón ${ }^{1,2,3}$, Angela Montenegro Cárdenas ${ }^{2,3}$, Joel Alberto Vargas Hernández ${ }^{1}$, Araceli Consuelo Hinojosa Juárez ${ }^{1}$

\section{Resumen}

Con el advenimiento de la terapia antirretroviral (ART), la infección por el virus de inmunodeficiencia humana (VIH) se ha convertido en una enfermedad crónica con complicaciones metabólicas importantes más acentuadas que en la población general. Mientras no se tenga una vacuna que erradique las tasas de infección y no exista una cura para esta pandemia, se debe ser más incisivo en el controlar las comorbilidades, entre las que destacan las alteraciones en el perfil de lípidos pues aumentan el riesgo cardiovascular.

Palabras claves: infección por el virus de inmunodeficiencia humana, terapia antirretroviral, perfil de lípidos, riesgo cardiovascular.

\begin{abstract}
With the advent of antiretroviral therapy (ART), infection with the human immunodeficiency virus (HIV) has become a chronic disease with major metabolic complications more pronounced than in the general population. While there is no vaccine to eradicate infection rates and there is no cure for this pandemic, it should be more incisive in controlling comorbidities, among which alterations in the lipid profile stand out as they increase cardiovascular risk.
\end{abstract}

Keywords: human immunodeficiency virus infection, antiretroviral therapy, lipid profile, cardiovascular risk.

1. Facultad de Medicina, Universidad Autónoma del Estado de México.

2. Asociación Científica Latina A.C. (ASCILA).

3. Ciprés Grupo Médico CGM.

Correspondencia: Dr. Hugo Mendieta Zerón. Felipe Villanueva sur 1209. Col. Rancho Dolores. C.P. 50170. Toluca, México.

Tel: 52-722-5410243. E-mail: drmendietaz@yahoo.com

ORCID

https://orcid.org/0000-0003-3492-8950 - Hugo Mendieta Zerón

https://orcid.org/0000-0002-4024-9252 - Angela Montenegro Cárdenas

https://orcid.org/0000-0001-7404-3132 - Joel Alberto Vargas Hernández

https://orcid.org/0000-0002-9100-9259 - Araceli Consuelo Hinojosa Juárez 


\section{Introducción}

El virus de inmunodeficiencia humana (VIH) provoca una falla progresiva del sistema inmunológico, que permite a las infecciones oportunistas poner en riesgo la vida de las personas que la padecen. Sin embargo, con el advenimiento de la terapia antirretroviral (ART), esta infección se ha convertido en una enfermedad crónica y las personas infectadas por el VIH ahora llevan una vida más larga y más saludable (1).

El tratamiento anti-VIH que se ofrece en la actualidad, una terapia retroviral altamente activa, ha permitido disminuir la morbilidad y la mortalidad tanto en países en desarrollo como desarrollados. No obstante este último logro, la terapia, que ha permitido un mejoramiento en la calidad de vida de los pacientes, a la par está provocando una problemática debido a los inhibidores de proteasas. Específicamente, la utilización de ART está generando las siguientes complicaciones metabólicas colaterales: dislipidemias, resistencia a la insulina, hipertensión, inflamación crónica, funciones alteradas del sistema inmune y diabetes mellitus; lo cual hace que se incrementen los riesgos cardiovasculares en los pacientes, así como su mortalidad $(2,3)$.

\section{Dislipidemia en VIH}

Es bien conocido que la infección por VIH, por sí sola, provoca cambios en funciones metabólicas. Por ejemplo, los pacientes infectados, antes de que surgieran los tra- tamientos, se reportaba que tenían las siguientes manifestaciones: decrementos en colesterol de alta densidad (HDL), colesterol de baja densidad (LDL) y colesterol total. Aumento en triglicéridos y ácidos grasos libres; lo que incrementa la susceptibilidad a la arteroesclerosis $(4,5,6)$.

Después de la implementación de la terapia antiretroviral convencional (HAART), que ayuda a reducir la morbilidad y mortalidad; al tiempo que maximiza la supresión del ARN viral, permite que se vaya recuperando y preservando la función inmune. Pero esta también ha provocado en los pacientes infectados la disminución de HDL, aumento de LDL, así como de triglicéridos (2). Con diferentes estudios se ha confirmado que la alteración del perfil lipídico ocasionada por los inhibidores de las proteasas se debe a que estos medicamentos provocan que el hígado aumente la producción lipídica, llevando al incremento del colesterol total, triglicéridos, LDL y lipoproteínas $(6,7)$. La aparición de la dislipidemia ocurre usualmente a los tres meses de haber iniciado con el tratamiento, y este tipo de dislipidemia está asociada al incremento en el riesgo de padecer arteroesclerosis (dislipidemia aterogénica).

Además de lo mencionado previamente, se ha reportado que existe una redistribución de la grasa en el cuerpo de las personas con VIH en tratamiento, caracterizada por una disminución de tejido adiposo en ciertas regiones del cuerpo como brazos, piernas o cara; mientras que en otras partes se va 
dando un aumento, como en zona hepática, cardiaca o intratorácica (8).

Existen varios factores que ayudan a paliar los efectos del empleo de la terapia retroviral y de las complicaciones propias de la infección, tales como cambios en los estilos de vida de los pacientes, particularmente ejercicio físico y dieta específica. De hecho, la modificación en las cantidades y tipos de lípidos que ingieren los pacientes ha mostrado un cambio significativo en la disminución del colesterol total en los pacientes con VIH (2).
La terapia retroviral, que usualmente se utiliza en los pacientes con VIH, es una combinación de tres clases de medicamentos: Inhibidores de transcriptasa inversa análogos de nucleósidos (NRTI), Inhibidores de transcriptasa inversa no análogos de nucleósidos (NNRTI), y los inhibidores de proteasas $(4,9)$. En la Tabla 1 se pueden observar los efectos en los perfiles lipídicos que producen los principales medicamentos empleados en la terapia retroviral para los pacientes infectados con VIH.

Tabla 1. Efecto global de los principales antirretrovirales en los perfiles lipídicos.

\begin{tabular}{|c|c|c|c|c|}
\hline Antiretroviral & Colesterol Total & LDL-C & HDL-C & Triglicéridos \\
\hline \multicolumn{5}{|l|}{ IPs (potenciados) } \\
\hline Lopinavir & $\boldsymbol{\uparrow} \uparrow$ & $\uparrow \uparrow$ & $\Leftrightarrow / \downarrow$ & $\uparrow \uparrow \uparrow$ \\
\hline Atazanavir & $\uparrow$ & $\Leftrightarrow / 1$ & $\Leftrightarrow /$ & $\Leftrightarrow$ \\
\hline Fosamprenavir & $\boldsymbol{\Lambda}$ & $\uparrow$ & $\Leftrightarrow /$ & $\boldsymbol{1} \boldsymbol{1}$ \\
\hline Saquinavir & $\boldsymbol{\uparrow} \uparrow$ & $\boldsymbol{\uparrow} \mathbf{1}$ & $\Leftrightarrow /$ & $\uparrow$ \\
\hline Darunavir & $\boldsymbol{\Lambda}$ & $\uparrow$ & $\Leftrightarrow / \nabla$ & $\boldsymbol{\Lambda}$ \\
\hline Tripanavir & $\boldsymbol{\uparrow} \uparrow$ & $\boldsymbol{1} \mathbf{1}$ & $\Leftrightarrow / \nabla$ & 1个1 \\
\hline \multicolumn{5}{|l|}{ NNRTIS } \\
\hline Efavirenz & $\boldsymbol{\Psi}$ & $\boldsymbol{\uparrow}$ & $\boldsymbol{1}$ & $\boldsymbol{1}$ \\
\hline Nevirapina & $\boldsymbol{1}$ & 1 & $\boldsymbol{\uparrow} \uparrow$ & $\Leftrightarrow \pi$ \\
\hline \multicolumn{5}{|l|}{ NRTIs } \\
\hline Tenofovir & $\Leftrightarrow / 1$ & $\Leftrightarrow / 1$ & $\Leftrightarrow / 1$ & \\
\hline Abacavir & $\Leftrightarrow / \hat{1}$ & $\uparrow$ & $\boldsymbol{1}$ & $\boldsymbol{\uparrow}$ \\
\hline Lamivudina & $\Leftrightarrow$ & $\Leftrightarrow$ & $\Leftrightarrow$ & $\Leftrightarrow$ \\
\hline Zidovudina & $\boldsymbol{1}$ & $\uparrow$ & $\boldsymbol{\uparrow}$ & $\boldsymbol{1} \boldsymbol{1}$ \\
\hline Stavudina & $\boldsymbol{\Lambda} \hat{\imath}$ & 11 & $\uparrow$ & $\boldsymbol{1} \uparrow$ \\
\hline \multicolumn{5}{|l|}{ Inhibidores de CCR5 } \\
\hline Maraviroc & $\Leftrightarrow$ & $\Leftrightarrow$ & $\Leftrightarrow / \uparrow$ & $\Leftrightarrow$ \\
\hline \multicolumn{5}{|l|}{ Inhibidores de Integrasa } \\
\hline Raltegravir & $\Leftrightarrow / \uparrow$ & $\Leftrightarrow / \uparrow$ & $\Leftrightarrow / \uparrow$ & $\Leftrightarrow$ \\
\hline
\end{tabular}

IPs: inhibidores de proteasas, NRTIs: inhibidores de los nucleósidos de transcriptasa inversa, NNRTIs: inhibidores de los no-nucleósidos de la transcriptasa inversa.

Fuente. Elaboración propia. 
En un estudio con pacientes infectados con VIH, se reportó que el valor del colesterol total subió por encima de los $240 \mathrm{mg} / \mathrm{dL}$ en el $27 \%$ de los pacientes con tratamiento exclusivo de inhibidores de proteasas, en 23\% de aquellos con tratamiento NNRTI, en $44 \%$ de quienes recibían una combinación de los anteriores y en 10\% de aquellos que sólo habían recibido tratamiento con NRTI; en contraste con el $8 \%$ en los que no recibieron tratamiento alguno. Además, se dio un incremento en triglicéridos por arriba de $200 \mathrm{mg} / \mathrm{dL}$ en el 40\% de pacientes con tratamiento con inhibidores de proteasas, en $32 \%$ de aquellos tratados con una combinación de inhibidores de proteasas y NNRTI, en 23\% en pacientes con NRTI y en $15 \%$ de aquellos que no recibieron tratamiento en absoluto (10).

Los avances en la tecnología de espectrometría de masas han facilitado la identificación detallada de los subtipos de lípidos individuales que en su conjunto aportan información valiosa para entender los mecanismos subyacentes al aumento del riesgo cardiovascular en pacientes con VIH. Por ejemplo, en un estudio por Boonthos y colaboradores usando espectrometría de masas por isonización en tándem por electropulverización, en participantes VIH positivos aleatorizados a uno de tres regímenes: 1) tenofovir/ emtricitabina con efavirenz, 2) atazanavir reforzado con ritonavir (atazanavir/r) o 3) zidovudina/abacavir, se encontró que el inicio de la terapia antirretroviral condujo a cambios significativos en el lipidoma que dependieron en parte del régimen aleatorio recibido. Esto dio lugar a diferencias significativas en 72 especies de lípidos y 7 clases (éster de colesterol, colesterol libre, fosfatidilcolina, gangliósido GM3, trihexosilceramida, monohexosilceramida y ceramidas) en la semana 48. Se observaron concentraciones de lípidos consistentemente más altas con efavirenz en comparación con atazanavir/r o zidovudina/abacavir. Doce de las especies de lípidos y dos clases de lípidos (ésteres de colesterol y ceramidas) que aumentaron significativamente en el brazo de efavirenz en comparación con los brazos de atazanavir/r o zidovudina/abacavir se han asociado previamente con futuros eventos cardiovasculares en pacientes VIH positivos. El cambio en la carga viral del VIH fue predictivo del cambio en 3 especies de lípidos. Se concluyó entonces que el inicio de la terapia antirretroviral condujo a cambios significativos en el lipidoma plasmático que fueron mayores en los que recibieron efavirenz (11)

\section{Tratamiento para la dislipidemia en VIH}

Los ensayos clínicos de resultados de las terapias para reducir el colesterol aún no se han completado en personas que viven con el VIH, por lo que las decisiones de tratamiento dependen de la extrapolación de los estudios en poblaciones no infectadas, lo cual dista de ser lo ideal. Un ejemplo claro es que aunque las estatinas son el pilar del tratamiento farmacológico hipolipemiante, deben considerarse las interacciones farmacológicas con el tratamiento antirretroviral, 
siendo así, la simvastatina y lovastatina están contraindicadas en pacientes que toman inhibidores de la proteasa, y la dosis de atorvastatina y rosuvastatina debe limitarse a 40 $\mathrm{mg} /$ día y $10 \mathrm{mg} /$ día, respectivamente, con algunas combinaciones de ART. Una estrategia útil para disminuir los efectos adversos sería sustituir las opciones más antiguas de ART por las más nuevas, siempre que se mantenga la supresión virológica, aunque siempre al agregar una estatina se reducirá LDL de manera más efectiva. Un aspecto agregado al valor del uso de las estatinas es que parecen ser protectoras del daño renal en pacientes portadores de VIH (12).

Aunado a lo anterior se ha demostrado que la Ezetimiba reduce el colesterol de lipoproteínas de baja densidad (LDL-C) sin interacciones medicamentosas con inhibidores de proteasas y estatinas. Además, la adición de ezetimiba a las estatinas es un tratamiento opcional en pacientes infectados por VIH con dislipidemia no controlada (13). Los estudios indican que las anormalidades de los lípidos no se tratan tan agresivamente en las personas que viven con el VIH como en las personas no infectadas, lo que hace que esta sea una oportunidad para mejorar la atención (14).

Ante la gravedad del riesgo aterogénico y cardiovascular en pacientes con VIH que reciben ART, se debe reforzar la estrategia en alimentación sana, pero a sabiendas de que esto no será suficiente para disminuir los niveles de lípidos se podría considerar el uso inclusive de medicina alternativa. Por ejemplo, se ha demostrado que el uso de un té que contiene Eryngium heterophyllum (hierba del sapo) y Amphipterygium adstringens (cuachalalate) diminuye los niveles de triglicéridos en un 30\% en población en general, y por lo tanto pudiera indicarse en pacientes con VIH de manera rutinaria ya que no presenta interacciones farmacológicas hasta el momento (15).

\section{Riesgo cardiovascular en pacientes con VIH}

Las personas con VIH muestran un aumento de la enfermedad cardiovascular (ECV), debido en parte al aumento de la activación inmune, la inflamación y la disfunción endotelial (16).

Las herramientas tradicionales de evaluación de riesgos subestiman el riesgo cardiovascular en personas con VIH. Por ejemplo, los estudios epidemiológicos en Europa y América del Norte han observado un cambio hacia un aumento de la incidencia de cardiopatías coronarias e infartos agudos de miocardio en poblaciones infectadas por VIH en comparación con la población general, incluso después de ajustar los factores de riesgo cardiovascular tradicionales (17). En la Tabla 2 se listan algunas de las tablas de riesgo cardiovascular que han sido utilizadas en pacientes con VIH. A la fecha se considera que el principal factor de riesgo es la hipertensión pero en realidad siempre hay que evaluar los factores individuales $(22,23)$. 
Tabla 2. Escalas de riesgo cardiovascular que han sido utilizadas en pacientes con VIH.

\begin{tabular}{|l|l|}
\multicolumn{1}{|c|}{ Escala de riesgo } & \multicolumn{1}{c|}{ Observaciones } \\
\hline The Framingham CVD calculator (15). & Subestima el riesgo en pacientes HIV positivos \\
\hline European Systematic Coronary Risk Evaluation score (SCORE) (16). & Poca concordancia con el DAD. \\
\hline $\begin{array}{l}\text { Data Collection on Adverse Effects of Anti-HIV Drugs Study (DAD) risk } \\
\text { equation (17). }\end{array}$ & El seguimiento de los pacientes es aún corto. \\
\hline QRISK2 calculator (18). & $\begin{array}{l}\text { Recomendada por la Asociación Británica de Pacientes que viven con } \\
\text { VIH. Subestima el riesgo cardiovascular. }\end{array}$ \\
\hline
\end{tabular}

Fuente. Elaboración propia.

Un problema importante de los pacientes con VIH es la distribución del tipo de grasa en el cuerpo (24). En pacientes con VIH / SIDA, especialmente aquellos con bajo recuento de CD4, se observa una lipodistrofia similar, que se llama síndrome de redistribución adiposa asociada al VIH (HARS) (25). El riesgo metabólico y cardiovascular de HARS ha sido estudiado previamente (26). Inicialmente se descubrió que estaba relacionado con los inhibidores de la proteasa, especialmente el indinavir, pero luego se demostró que también estaba relacionado con otra clase de fármacos antirretrovirales, especialmente los NRTIs, como lamivudina, estavudina y didanosina. La lipoatrofia (la pérdida de grasa subcutánea de cara, brazo, piernas, abdomen y glúteos) se asocia comúnmente con NRTIs (27) mientras que la lipohipertrofia (la acumulación de grasa subcutánea en la parte superior de la espalda, el cuello, el tronco y alrededor de las vísceras abdominales) se ve comúnmente con inhibidores de la proteasa (28). La inhibición de la polimerasa del ADN mitocondrial (ADNmt) por los NRTI es la razón del desarrollo de la lipoatrofia y otras toxicidades. Cambiar los medicamentos dentro de la clase de NRTIs con menor toxicidad de ADNmt podrá limitar o revertir la lipoatrofia (29).

\section{Comorbilidades agregadas a dislipidemia}

En un estudio en Puerto Rico, donde se revisaron un total de 250 (179 hombres; 71 mujeres) registros médicos, con un edad media de los participantes de 47.9 ańos y, en promedio, habiendo estado viviendo con el VIH durante 9 ańos, la mayoría (97.6\%) tenía al menos una comorbilidad, siendo la dislipidemia e hipertensión las más comunes (30).

En un estudio en Italia, en el que se incluyeron 1087 pacientes (edad media $47.9 \pm$ 10.8), 190 (17.5\%) no tenían comorbilidad, mientras que $285(26.2 \%)$ tenían una condición y 612 (56.3\%) eran multimórbidos. Las asociaciones más recurrentes fueron: 1) dislipidemia + hipertensión $(237,21.8 \%)$; 2) dislipidemia + EPOC (188, 17.3\%); 3) $\mathrm{EPOC}+\mathrm{VHC}-\mathrm{Ab}+(141,12.9 \%)$. La morbilidad múltiple se asoció con la edad avanzada, un índice de masa corporal más alto, 
tabaquismo actual o previo, el estadio C de los CDC y una mayor duración del TAR (31).

\section{VIH en la tercera edad}

El tratamiento antirretroviral de gran actividad (HAART por sus siglas en inglés), generalmente permite el control de la infección por VIH. Sin embargo, las personas afectadas por este virus, cuando envejecen, presentan una alta prevalencia de enfermedades metabólicas (32). Es así que, el envejecimiento en pacientes con VIH en se caracteriza por una incidencia creciente de enfermedades crónicas no trasmisibles y polifarmacia. En un estudio en Brasil la dislipidemia fue la comorbilidad más frecuente seguida de diabetes y osteoporosis. A su vez, el uso de medicamentos concomitantes para el tratamiento de las enfermedades crónicas no trasmisibles aumentó significativamente entre la primera y la última visita, especialmente los antihipertensivos, los hipolipemiantes y los medicamentos para la diabetes (33).

\section{Embarazo}

El embarazo es un estado fisiológico que induce la hipertrigliceridemia (34). En el caso de mujeres gestantes con VIH, el uso de la ART en el embarazo presenta beneficios incuestionables en la prevención de la transmisión maternoinfantil (TMI) del VIH, aunque se asocia con efectos adversos maternos. En una cohorte en Francia de 793 embarazos, con una tasa de TMI fue de
$2.3 \%$, con $0.8 \%$ en los últimos 5 ańos, los efectos adversos maternos fueron: dislipidemia (82\%), anemia (56\%), anomalías en las pruebas de función hepática $(54.5 \%)$, incluyendo hiperbilirrubinemia (11.6\%), alteración de la glucemia en ayunas (19.2\%), trombocitopenia $(14.1 \%)$ y reacción alérgica $(2.7 \%)$. La mayoría de los efectos adversos considerados relacionados con ART en este estudio fueron leves según la escala DAIDS (The Division of AIDS Table for Grading the Severity of Adult and Pediatric Adverse Events). En el análisis multivariado, las coinfecciones y el inicio de TAR durante el embarazo fueron factores de riesgo de anemia materna, mientras que el recuento de CD4 superior a 200 células/mm3 fue protector. Los regímenes de nevirapina, nelfinavir y atazanavir aumentaron el riesgo de anomalías en las pruebas de función hepática mientras que el uso de lopinavir durante el embarazo aumentó el riesgo de alteración de la glucemia en ayunas (35).

\section{Niños}

Siguiendo con lo anterior, es alarmante la alta incidencia de casos de niños infectados con VIH, lo que hace que también presenten dislipidemia al iniciar el ART (36) y se registran a su vez casos de cardiopatía a temprana edad (37). Esta situación hace extremadamente importante que se lleve a cabo una evaluación sistemática del riesgo cardiovascular y la detección de enfermedades en niños infectados con VIH que reciben terapia antirretroviral (38). 
Se puede recomendar la práctica de actividad física constante ya que los altos niveles de actividad física moderada a vigorosa y aeróbica pueden prevenir el desarrollo de factores de riesgo cardiovascular en niños y adolescentes VIH+ (39-40).

\section{Expectativas futuras}

El análisis de las bases de datos disponibles relacionadas con el VIH / SIDA confirma un cambio de paradigma en la esperanza de vida del paciente: ahora el VIH se ha convertido en una enfermedad crónica, por lo que los pacientes están envejeciendo. Sin embargo, este avance está acompańado por una contraparte negativa: debido al aumento en el número de ańos de vida ganados, existe una prevalencia de comorbilidades mayor que la población general y en una edad más temprana. Reducir el riesgo asociado con todas las comorbilidades que puede desarrollar el paciente anciano con VIH / SIDA, ahora debe ser un objetivo de salud (41).

A raíz de los conocimientos vigentes se determina el mayor riesgo aterogénico y cardiovascular de la población con VIH en relación a la población no afectada por esta enfermedad, de tal manera que es imperativo llevar un control estricto del perfil lipídico para instaurar el mejor tratamiento.

Está aún en seguimiento el estudio de los Inhibidores de proproteína convertasa subtilisina/kexina tipo 9 (iPCSK9) en pacientes $\mathrm{VIH}+\mathrm{y}$ con dislipidemia pero no se recomienda su uso a largo plazo $(42,43)$.
Los cambios que se esperarían a futuro, serían una generación de nuevos antiretrovirales con menos efectos deletéreos metabólicos (44), pero quizás, lo que esperamos todos es que se tenga éxito en el desarrollo de alguna vacuna, para lo cual los 2 enfoques actuales principales para la generación de inmunidad protectora son a través de anticuerpos ampliamente neutralizantes (bnAb) e inducción de anticuerpos funcionales (Abs no neutralizantes con otras posibles funciones antivirales) (45) pues sería una solución masiva a diferencia del trasplante de células madre (46).

\section{Referencias}

1. Ahmed D, Roy D, Cassol E. Examining Relationships between Metabolism and Persistent Inflammation in HIV Patients on Antiretroviral Therapy. Mediators Inflamm. 2018 Sep 27;2018:6238978.

2. Andrade MA, Andrade HA. Alteraciones del metabolismo lipídico en pacientes seropositivos para VIH con tratamiento Retroviral altamente efectivo. Rev. Cuban Cardiol Circ Cardiovasc. 2010;16(1):64-73.

3. Batterham MJ, Brown D, Workman C. Modifying dietary fat intake can reduce serum cholesterol in HIV-associated hypercholesterolemia. AIDS. 2003;17(9):1414-1416.

4. Castelo Elías-Calles L, González Calero TM. Dislipidemia y virus de inmunodeficiencia adquirida/ SIDA. Rev. cuba endocrinol. 2010;21(2):202222 .

5. Friis-Møller N, Weber R, Reiss P, Thiébaut R, Kirk O, d'Arminio Monforte A, et al; DAD study group. Cardiovascular disease risk factors in HIV patients--association with antiretroviral therapy. Results from the DAD study. AIDS. 2003;17(8):11791193. 
6. Grinspoon S, Carr A. Cardiovascular risk and bodyfat abnormalities HIV-infected adults. N Engl J Med. 2005;352(1):48-62.

7. Pao V, Lee GA, Grunfeld C. HIV therapy, metabolic syndrome, and cardiovascular risk. Curr Atheroscler Rep. 2008;10(1):61-70.

8. Rodríguez-Carranza SI, Aguilar-Salinas CA. Anormalidades metabólicas en pacientes con infección por VIH. Rev Invest Clin. 2004;56(2):193-208.

9. Husain NE, Ahmed MH. Managing dyslipidemia in HIV/AIDS patients: challenges and solutions. HIV AIDS (Auckl). 2014;7:1-10.

10. Feeney ER, Mallon PW. HIV and HAART-Associated Dyslipidemia. Open Cardiovasc Med J. 2011;5:49-63.

11. Boonthos K, Puttilerpong C, Pengsuparp T, Manosuthi W. Short-Term Efficacy and Safety of Adding Ezetimibe to Current Regimen of Lipid-Lowering Drugs in Human Immunodeficiency Virus-Infected Thai Patients Treated with Protease Inhibitors. Jpn J Infect Dis. 2018;71(3):220-224.

12. Calza L, Colangeli V, Borderi M, Manfredi R, Marconi L, Bon I, Re MC, Viale P. Rosuvastatin and atorvastatin preserve renal function in HIV-1-infected patients with chronic kidney disease and hyperlipidaemia. HIV Clin Trials. 2018;19(3):120-128.

13. Leyes P, Martínez E, Larrousse M, Cofán M, Trabal J, Pérez-Heras AM, Forga MT, Ros E. Effects of ezetimibe on cholesterol metabolism in HIV-infected patients with protease inhibitor-associated dyslipidemia: a single-arm intervention trial BMC Infect Dis. $2014 ; 14: 497$.

14. Waters DD, Hsue PY. Lipid Abnormalities in Persons Living With HIV Infection. Can J Cardiol. 2019;35(3):249-259.

15. García Gómez Y, Mendieta Alcántara MR, Mendieta Zerón H. Eryngium heterophyllum and Amphipterygium adstringens Tea Effect on Triglyceride Levels: A Clinical Trial. Trad Integr Med. 2019;4(1):22-27.
16. Toribio M, Fitch KV, Stone L, Zanni MV, Lo J, de Filippi C, Sponseller CA, Lee H, Grundberg I, Thompson MA, Aberg JA, Grinspoon SK. Assessing statin effects on cardiovascular pathways in HIV using a novel proteomics approach: Analysis of data from INTREPID, a randomized controlled trial. EBioMedicine. 2018;35:58-66.

17. Strijdom H, De Boever P, Walzl G, Essop MF, Nawrot TS, Webster I, et al. Cardiovascular risk and endothelial function in people living with HIV/ AIDS: design of the multi-site, longitudinal EndoAfrica study in the Western Cape Province of South Africa. BMC Infect Dis. 2017;17(1):41.

18. D’Agostino RB Sr, Grundy S, Sullivan LM, Wilson P; CHD Risk Prediction Group. CHD Risk Prediction Group. Validation of the Framingham coronary heart disease prediction scores: results of a multiple ethnic groups investigation. JAMA. 2001;286(2):180-187.

19. Begovac J, Dragović G, Višković K1, Kušić J, Perović Mihanović M, Lukas D, et al. Comparison of four international cardiovascular disease prediction models and the prevalence of eligibility for lipid lowering therapy in HIV infected patients on antiretroviral therapy. Croat Med J. 2015;56(1):14-23.

20. Friis-Møller N, Thiébaut R, Reiss P, Weber R, Monforte $\mathrm{AD}, \mathrm{De} W \mathrm{~W} \mathrm{~S}$, et al; DAD study group. Predicting the risk of cardiovascular disease in HIVinfected patients: the data collection on adverse effects of anti-HIV drugs study. Eur J Cardiovasc Prev Rehabil. 2010;17(5):491-501.

21. Hippisley-Cox J, Coupland C, Vinogradova Y, Robson J, Minhas R, Sheikh A, et al. Predicting cardiovascular risk in England and Wales: prospective derivation and validation of QRISK2. BMJ. 2008 Jun 28;336(7659):1475-1482.

22. Pierre S, Seo G, Rivera VR, Walsh KF, Victor JJ, Charles B, Julmiste G, Dumont E, Apollon A, Cadet M, Saint-Vil A, Marcelin A, Severe P, Lee MH, Kingery J, Koenig S, Fitzgerald D, Pape J, McNairy ML. 
Prevalence of hypertension and cardiovascular risk factors among long-term AIDS survivors: A report from the field. J Clin Hypertens (Greenwich). 2019 Aug 26. doi: 10.1111/jch.13663.

23. Pushpakom S, Kolamunnage-Dona R, Taylor C, Foster T, Spowart C, García-Fiñana M, Kemp GJ, Jaki T, Khoo S, Williamson P, Pirmohamed M; TAILoR Study Group TAILoR (TelmisArtan and InsuLin Resistance in Human Immunodeficiency Virus [HIV]): An Adaptive-design, Dose-ranging Phase IIb Randomized Trial of Telmisartan for the Reduction of Insulin Resistance in HIV-positive Individuals on Combination Antiretroviral Therapy. Clin Infect Dis. 2019 Jul 3. pii: ciz589. doi: 10.1093/ cid/ciz589.

24. Kumar S, Dhanwal DK. Central obesity \& dyslipidemia in HIV patients on antiretroviral therapy. Indian J Med Res. 2018;148(4):366-368.

25. Lichtenstein K, Balasubramanyam A, Sekhar R, Freedland E. HIV-associated adipose redistribution syndrome (HARS): definition, epidemiology and clinical impact. AIDS Res Ther. 2007;4:16.

26. Balasubramanyam A, Sekhar RV, Jahoor F, Jones PH, Pownall HJ Pathophysiology of dyslipidemia and increased cardiovascular risk in HIV lipodystrophy: a model of 'systemic steatosis'Curr Opin Lipidol. 2004;15(1):59-67.

27. Margolis AM, Heverling H, Pham PA, Stolbach A. A review of the toxicity of HIV medications. J Med Toxicol. 2014;10(1):26-39.

28. Lake JE, McComsey GA, Hulgan TM, Wanke CA, Mangili A, Walmsley SL, Boger MS, Turner RR, McCreath HE, Currier JS. A randomized trial of Raltegravir replacement for protease inhibitor or non-nucleoside reverse transcriptase inhibitor in HIV-infected women with lipohypertrophy. AIDS Patient Care STDS. 2012;26(9):532-40.

29. Rojas J, Lonca M, Imaz A, Estrada V, Asensi V, Miralles $\mathrm{C}$, Domingo P, Montero M, del Rio L, Fontdevila J, Perez I, Cruceta A, Gatell JM, Arnedo M, Martínez E. Improvement of lipoatrophy by swit- ching from efavirenz to lopinavir/ritonavir. HIV Med. 2016;17(5):340-9.

30. Rodríguez-Díaz CE, Santiago-Rodríguez EI, Jovet-Toledo GG, Santana-Bagur J, Ron-Suarez Y, Orengo JC, Arbelaez F, Monsanto H. Comorbidities in a sample of adults with HIV in Puerto Rico: an exploratory study. HIV AIDS (Auckl). 2019;11:155-164.

31. Maggi P, Santoro CR, Nofri M, Ricci E, De Gennaro N, Bellacosa C, Schiaroli E, Orofino G, Menzaghi B, Di Biagio A, Squillace N, Francisci D, Vichi F, Molteni C, Bonfanti P, Gaeta GB, De Socio GV. Clusterization of co-morbidities and multi-morbidities among persons living with HIV: a cross-sectional study. BMC Infect Dis. 2019;19(1):555.

32. Lagathu C, Béréziat V, Gorwood J, Fellahi S, Bastard JP, Vigouroux C, Boccara F, Capeau J. Metabolic complications affecting adipose tissue, lipid and glucose metabolism associated with HIV antiretroviral treatment. Expert Opin Drug Saf. 2019;18(9):829-840.

33. Brites C, Nogueira RS, Gosuen GC, Kalmar EMN, Leme ST, Martins RT, Oyama T, Bastos F. Getting older with HIV: increasing frequency of comorbidities and polypharmacy in Brazilian HIV patients. AIDS Res Hum Retroviruses. 2019 Aug 27. doi: 10.1089/AID.2019.0069. [Epub ahead of print]

34. Mendieta-Zerón H, Huerta-Coyote O. Dyslipidemia is a persistent problem in puerperium with or without preeclampsia. Clin Exp Obstet Gynecol. 2013;40(2):229-32

35. Delicio AM, Lajos GJ, Amaral E, Lopes F, Cavichiolli F, Myioshi I, Milanez H. Adverse effects of antiretroviral therapy in pregnant women infected with HIV in Brazil from 2000 to 2015: a cohort study. BMC Infect Dis. 2018;18(1):485.

36. Smith C, McFarland EJ. Update on Pediatric Human Immunodeficiency Virus Infection: Paradigms in Treatment and Prevention. Adv Pediatr. 2016;63(1):147-71. 
37. Manafe N, Ngale A, Biquiza N, Zimba I, Majid N, Mocumbi AO. Need for active cardiovascular screening in HIV-infected children under antiretroviral therapy in Africa. Cardiovasc Diagn Ther. 2019;9(1):68-72.

38. Arrive E, Viard JP, Salanave B, Dollfus C, Matheron S, Reliquet V, Arezes E, Nailler L, Vigouroux C, Warszawski J; ANRS CO19 COVERTE and ENNS study groups. Metabolic risk factors in young adults infected with HIV since childhood compared with the general population. PLoS One. 2018;13(11):e0206745

39. de Lima LRA, Back IC, Nunes EA, Silva DAS, Petroski EL. Aerobic fitness and physical activity are inversely associated with body fat, dyslipidemia and inflammatory mediators in children and adolescents living with HIV. J Sports Sci. 2019;37(1):50-58.

40. Zárate Sanabria, Andrés Gabriel et al. Intervención educativa sobre virus de inmunodeficiencia humana en la comunidad habitante de calle que asiste a un centro de acogida en la ciudad de Bogotá D.C. Colombia. Nova, [S.1.], v. 15, n. 28, p. 11 - 17, oct. 2017. ISSN 2462-9448. Disponible en: <http:// hemeroteca.unad.edu.co/index.php/nova/article/ view/2072>. Fecha de acceso: 17 may. 2019 doi:https://doi.org/10.22490/24629448.2072.

41. Del Amo J, Campbell C, Navarro G, Segura F, Suárez I, Teira R, et al. HIV in Spain 2017: policies for a new management of chronicity beyond virological control]. Rev Esp Salud Publica. 2018 Sep 6;92. pii: e201809062.

42. Kohli M, Patel K, MacMahon Z, Ramachandran R, Crook MA, Reynolds TM, Wierzbicki AS. Pro-protein subtilisin kexin-9 (PCSK9) inhibition in practice: lipid clinic experience in 2 contrasting UK centres. Int J Clin Pract. 2017 Nov;71(11). doi: 10.1111/ijcp.13032. Epub 2017 Oct 10

43. Ruscica M, Watts GF, Sirtori CR. PCSK9 in HIV infection: New opportunity or red herring? Atherosclerosis. 2019;284:216-217
44. Nawej Tshikung O, Calmy A. [Antiretroviral treatments: towards simplified regimens? Rev Med Suisse. 2018;14(588-589):102-106.

45. Hsu DC, O'Connell RJ. Progress in HIV vaccine development. Hum Vaccin Immunother. 2017;13(5):1018-1030.

46. Zou S, Glynn S, Kuritzkes D, Shah M, Cook N, Berliner N; NHLBI AIDS Blood Session Working Group. NHLBI AIDS Blood Session Working Group. Hematopoietic cell transplantation and HIV cure: where we are and what next? Blood. 2013;122(18):3111-3115. 